Frontal subcortial white matter lesions following PFS with encephalopathy are described in a diffusion-weighted MRI study between 3 and 9 days after the seizure. Diffusion abnormalities disappeared between days 9 and 25, leaving cerebral atrophy after 2 weeks (Takanashi J et al. Neurology 2006;66:1304-1309; Ped Neur Briefs May 2006;20:33).

\title{
ETIOLOGY, EEG, AND MRI FINDINGS IN NONCONVULSIVE SEIZURES WITH IMPAIRED CONSCIOUSNESS
}

Nonconvulsive seizures (NCS) in children admitted to the Pediatric Intensive Care Unit (PICU) from Jan 2000 to Dec 2003 were analyzed in a study at The Hospital for Sick Children, Toronto, Ont, Canada. A NCS was defined as a deterioration of consciousness accompanied by at least one episode of electrographic seizure lasting longer than $10 \mathrm{~s}$. Of 141 patients admitted with unexplained altered consciousness, no overt seizure, and EEG recording within $24 \mathrm{~h}, 23(16.3 \%)$ had NCS. Male to female ratio was 1.9:1. The mean age was 40.1 mo (range: 1 mo to $18 \mathrm{y}$ ); infants $<1$ mo were excluded. No preexisting disease was present in $10(43 \%) ; 8(35 \%)$ had a history of seizures, $2(9 \%)$ congenital heart disease, and 3 $(13 \%)$ other underlying disease. None had nonconvulsive status epilepticus. The etiology of NCS was an acute structural brain lesion in 11 (48\%); cerebral infarction in 6, subdural hematoma in 3, cerebral hemorrhage in 1, and AV malformation in 1. Other etiologies included meningitis in $3(13 \%)$, seizure disorder in $3(13 \%)$, sepsis in 2 and pneumonia in 2. EEG showed right-sided epileptiform discharges in $9(39.2 \%)$, most commonly temporal; the EEG pattern was S/W in $9(39.1 \%)$. The mean duration of EEG discharge was $159 \mathrm{~s}$ (range: $10 \mathrm{~s}$ to $11 \mathrm{~min})$. MRI or CT was abnormal in $18(78.2 \%)$, most commonly cerebral infarction in $6(26.1 \%)$, followed by diffuse edema in $5(21.7 \%)$. Brain imaging and EEG were concordant in localization in $10(55.6 \%)$. All seizures responded to treatment with IV phenobarbital or phenytoin. (Saengpattrachai M, Sharma R, Hunjan A et al. Nonconvulsive seizures in the pediatric intensive care unit: Etiology, EEG, and brain imaging findings. Epilepsia Sept 2006;47:1510-1518). (Reprints: Dr MA Cortez, Division Neurology, Department of Pediatrics, The Hospital for Sick Children, 555 University Ave, Toronto, Ontario M5G 1X8, Canada).

COMMENT. Of children admitted to the PICU with altered state of consciousness, $16 \%$ have NCS. A higher prevalence (33\%) reported in a previous study is explained by the inclusion of neonates and PLEDs. In contrast to adults who often have preexisting epilepsy and traumatic brain injury as precursors of NCS, children have no preexisting disease in $43 \%$ of cases, cerebral infarction as the most common NCS etiology, and a predominance of right hemisphere localization. An early EEG is indicated in children with deterioration of consciousness of uncertain origin.

Nonconvulsive status epilepticus is described in 19 pediatric patients admitted to the Epilepsy Center, Columbia University, NY, June 2000 - Dec 2003. Five developed NCSE following convulsive SE and 12 followed brief convulsions. Acute hypoxic-ischemic injury was the most frequent etiology (in $5(26 \%)$ ). Six had associated periodic lateralized epileptiform discharges (PLEDs), and 1 had generalized periodic epileptiform discharges (GPEDs), both associated with worse outcome. Five $(26 \%)$ died of the underlying acute 
medical illness. Prolonged EEG monitoring may be warranted after brief convulsive seizures to exclude NCSE. (Tay SKH, Hirsch LJ, Leary L et al. Epilepsia Sept 2006;47:1504-1509).

\section{PANAYIOTOPOULOS SYNDROME: BENIGN AUTONOMIC EPILEPSY}

The clinical features, EEG, misdiagnoses, and management of children with Panayiotopoulos syndrome (PS) are reviewed from Sophia Children's Hospital, GoudiAthens, Greece. Emesis is the predominant manifestation of autonomic seizures in PS. Other autonomic symptoms include pallor, mydriasis, urinary or fecal incontinence, and hypersalivation. One fifth have ictal syncope with or without convulsions. One half of the seizures last for $>30$ minutes, and constitute a nonconvulsive autonomic status. Two thirds of the seizures occur during sleep. The EEG shows multiple spikes, occipital predominating, and sometimes centro-temporal of Rolandic epilepsy. Prognosis is benign, one quarter of patients having a single seizure and half having 2 to 5 seizures. One fifth develop Rolandic or occipital seizures, remitting before age 16 years. Cardiorespiratory arrest during an autonomic seizure is a very rare occurrence. Misdiagnoses include acute encephalitis, syncope, migraine, benign Rolandic epilepsy, or gastroenteritis. Benzodiazepines are used to terminate nonconvulsive autonomic status, and prophylactic treatment is prescribed infrequently. Aggressive treatment is avoided because of risk of cardiorespiratory arrest. (Covanis A. Panayiotopoulos syndrome: a benign childhood autonomic epilepsy frequently imitating encephalitis, syncope, migraine, sleep disorder, or gastroenteritis. Pediatrics October 2006;118:1237-1243). (Respond: A Covanis MD, Neurology Department, Agia Sophia Children's Hospital, Thivon and Levadias, Goudi, 11527 Athens, Greece).

COMMENT. Vomiting as an ictal phenomenon has been described by various terms including ictus emeticus, diencephalic or autonomic epilepsy, abdominal epilepsy, and cyclic vomiting as a form of epilepsy (Millichap JG, Lombroso CT, Lennox WG. Pediatrics 1955;15:705-714; Freeman R, Schachter SC. Seminars in Neurology 1995;15:158-166; Ped Neur Briefs June and August 1995). Occipito-temporal spikes in the EEG, similar to those mentioned with PS, are described in previous reports of ictal vomiting. Are the symptoms described as PS sufficiently specific and novel to warrant classification as a separate syndrome?

\section{ANTIEPILEPTIC DRUGS}

\section{OXCARBAZEPINE MONOTHERAPY}

The efficacy of oxcarbazepine monotherapy in 60 children and adolescents (aged 6 months to 17.8 years; mean age 8.2 yrs) with partial onset epilepsy was evaluated by retrospective chart review in a study at St Christopher's Hospital for Children, Philadelphia, PA. Dosage ranged from 6 to $71 \mathrm{mg} / \mathrm{kg} /$ day (mean $26.3 \mathrm{mg} / \mathrm{kg} /$ day). The mean dose varied with age, higher doses were required in younger patients; in patients $<4$ years, the mean dose was $33.1 \mathrm{mg} / \mathrm{kg} /$ day; $8-12$ years, $25 \mathrm{mg} / \mathrm{kg} /$ day. Duration of therapy was 3 months to 8 years (mean duration 16.7 months). Reduction in seizure frequency was $>50 \%$ in $51(85 \%)$ 